\title{
Hydrogen-rich saline regulates the polarization and apoptosis of alveolar macrophages and attenuates lung injury via suppression of autophagy in septic rats
}

\author{
Peng Qiu ${ }^{1}$, Yang Liu ${ }^{2}$, Keyan Chen ${ }^{3}$, Youjing Dong ${ }^{1}$, Shiqing Liu ${ }^{1}$, Jin Zhang ${ }^{1}$ \\ ${ }^{1}$ Department of Anesthesiology, Shengjing Hospital of China Medical University, Shenyang, China; ${ }^{2}$ Department of Oncology, Shengjing Hospital \\ of China Medical University, Shenyang, China; ${ }^{3}$ Department of Laboratory Animal Science, China Medical University, Shenyang, China \\ Contributions: (I) Conception and design: P Qiu, J Zhang; (II) Administrative support: None; (III) Provision of study materials or patients: P Qiu, \\ K Chen; (IV) Collection and assembly of data: S Liu, Y Dong; (V) Data analysis and interpretation: Y Liu, K Chen; (VI) Manuscript writing: All \\ authors; (VII) Final approval of manuscript: All authors. \\ Correspondence to: Jin Zhang. Department of Anesthesiology, Shengjing Hospital of China Medical University, No. 36 Sanhao St., Shenyang 110004, \\ China. Email: zhangj_sj@163.com.
}

\begin{abstract}
Background: Hydrogen-rich saline (HRS) has a protective effect on sepsis-induced lung injury. However, the underlying mechanisms are still unclear. Polarization and apoptosis of macrophages are essential factors in the pathogenesis of acute lung injury (ALI). Moreover, autophagy is involved in the regulation of both macrophage polarization and apoptosis. Therefore, this study investigated the ability of HRS to attenuate ALI through regulation of the polarization and apoptosis of alveolar macrophages (AMs) during sepsis by modulating autophagy.

Methods: Male Sprague-Dawley (SD) rats were used to prepare the sepsis-induced lung injury animal model. Rat lung tissue was harvested after lipopolysaccharide (LPS) treatment, in the presence or absence of HRS, and the AMs were analyzed for changes in polarization, apoptosis, and autophagy. The rat AM cell line NR8383 was used to examine these processes in vitro using Western blot analysis, flow cytometry, and transmission electron microscopy.

Results: LPS-induced ALI in rats was associated with an increase in autophagy, apoptosis, and M1 polarization but a decrease in M2 polarization in AMs. These effects were reversed by administration of HRS. Inhibition of AM autophagy with 3-methyladenine (3-MA) decreased apoptosis and M1 polarization and increased M2 polarization, paralleling the effects of HRS.

Conclusions: HRS could attenuate ALI in septic rats through regulation of AM polarization and a reduction in apoptosis by suppressing autophagy. This may represent a potential novel therapeutic target for the treatment of ALI caused by sepsis.
\end{abstract}

Keywords: Hydrogen-rich saline (HRS); acute lung injury (ALI); macrophage; autophagy; apoptosis; polarization

Submitted Apr 02, 2021. Accepted for publication Jun 15, 2021.

doi: 10.21037/atm-21-2489

View this article at: https://dx.doi.org/10.21037/atm-21-2489

\section{Introduction}

Acute lung injury (ALI) and acute respiratory distress syndrome (ARDS) are common causes of death in patients with sepsis (1). ALI is characterized by severe hypoxemia, respiratory insufficiency, and pulmonary edema (2). Pathological changes include alveolar-capillary barrier damage, exudation of protein-rich liquid, alveolar macrophage (AM) activation, neutrophil infiltration, and cellular apoptosis and necrosis (3). Unfortunately, there are very few effective treatments for these conditions. Recent studies suggested that hydrogen-rich saline (HRS), a natural selective antioxidant (4), has a protective effect against ALI in animal models of sepsis (5-8). However, the potential mechanisms of action are not fully understood. 
ALI from sepsis is usually attributed to uncontrolled systemic inflammation rather than mediated by reactive oxygen species (ROS). Therefore, the protective effects of HRS on the lungs may relate to mechanisms other than anti-oxidation. Previous studies have established that HRS exerts biological effects not only by scavenging ROS, but also by affecting gene expression and signal transduction (9).

Early over-activation and late suppression of the immune system are core mechanisms underlying the development of sepsis, in which macrophages play a crucial role (10). Recent studies have demonstrated that macrophages are key factors in the pathogenesis of ALI and ARDS $(2,11)$. Therefore, we hypothesized that regulation of macrophage function by HRS is a potential mechanism of lung protection during sepsis, however, and this has not been previously investigated. There are two phenotypes of macrophage activation, namely, M1 and M2 polarization (12). The main phenotype of resident AMs, under normal physiological conditions, is M2 (2). When infections occur, resident $\mathrm{M} 2$ and recruited macrophages switch to M1 and eliminate the pathogens by releasing a large number of pro-inflammatory mediators and by recruiting neutrophils (2). During sepsis, excessive M1 polarization leads to uncontrolled inflammation and lung injury, whereas M2 polarization restricts the inflammatory response and promotes tissue repair $(2,10)$. In addition, apoptosis of macrophages contributes to immune suppression and exacerbates infections (10). Therefore, this study investigated the effects of HRS on the polarization and apoptosis of AMs.

Autophagy has been shown to play an essential role in immunity and inflammation $(13,14)$. It is an important means of maintaining homeostasis and cell renewal involved in the regulatory mechanisms of both macrophage polarization and apoptosis (15-17). Thus, regulating autophagy in macrophages might be a promising therapeutic strategy against ALI and ARDS in sepsis. The present study investigated the hypothesis that HRS could attenuate sepsis-induced ALI through regulation of macrophage polarization and inhibition of macrophage apoptosis by modulating autophagy.

We present the following article in accordance with the ARRIVE reporting checklist (available at https://dx.doi. org/10.21037/atm-21-2489).

\section{Methods}

\section{Ethics statement}

All animal studies were performed with the approval of the China Medical University Laboratory Animal Welfare and Ethics Committee (IACUC No. 2015048R) in accordance with the National Institutes of Health (Bethesda, MD, USA) guidelines for the care and use of laboratory animals. A protocol was prepared before the study without registration.

\section{Animals and cells}

Male Sprague-Dawley (SD) rats (aged 8-10 weeks and weighing 300-350 g) were purchased from the Experimental Animal Center of China Medical University (Shenyang, China). We only included male rats to minimize the potential confounders which could affect the study results, including effects of oestrogen. Rat were all healthy detected by Veterinary. Rats were housed in individual cages under a $12 \mathrm{~h} / 12 \mathrm{~h} \mathrm{light} /$ dark cycle at $22 \pm 2{ }^{\circ} \mathrm{C}$ and $50 \% \pm 5 \%$ relative humidity, with free access to drink and food. The NR8383 rat AMs were purchased from the Chinese Academy of Sciences cell bank (Shanghai, China).

\section{HRS preparation}

The HRS was prepared as previously described (18). Briefly, hydrogen was dissolved in normal saline (NS) under high pressure $(0.4 \mathrm{MPa})$ for 6 hours to reach supersaturation and then stored under atmospheric pressure at $4{ }^{\circ} \mathrm{C}$ in an aluminum bag with no dead volume. The HRS was prepared weekly to ensure that the $\mathrm{H}^{+}$concentration was maintained above $0.6 \mathrm{mmol} / \mathrm{L}$. Each solution was sterilized by $\gamma$-irradiation. The average HRS concentration was $0.8 \mathrm{mmol} / \mathrm{L}$, as measured by gas chromatography (4).

\section{Sepsis-induced lung injury model and HRS treatment}

Rats were fasted for 12 hours prior to treatment but given free access to water. A total of $96 \mathrm{SD}$ rats were randomly (random number table method) divided into 4 groups $(n=24)$, no a priori sample size calculation was performed before the study. Authors were aware of group allocation at the different stages of the experiment. The four groups were named as the control group, the HRS group, the lipopolysaccharide (LPS) group, and the LPS + HRS group. In the LPS and LPS + HRS groups, sepsis-induced ALI was established by the intravenous administration of $5 \mathrm{mg} / \mathrm{kg}$ LPS. No rats died or needed to be terminated due to LPS injection. Rats inoculated with LPS were considered as inflammatory controls. There were no exclusions. In the LPS + HRS group, rats were intraperitoneally (ip) 
administered $10 \mathrm{~mL} / \mathrm{kg}$ HRS 1 hour after sepsis-induced ALI (19). Rats in the control and HRS groups were treated with NS ip and HRS $(10 \mathrm{mg} / \mathrm{kg}$, ip), respectively, without LPS treatment. At different time points after drug administration, rats were anesthetized with $2 \%$ pentobarbital sodium and euthanized by exsanguination via the abdominal aorta. The lung tissue was harvested at 4, 8 , 12 , and 24 hours after treatment to assess the histological changes ( $\mathrm{n}=6$ rats per group per time point). One portion of lung tissue from each rat was fixed in $10 \%$ formaldehyde and the remainder was preserved at $-80^{\circ} \mathrm{C}$.

\section{In vitro evaluation of HRS in NR8383 AM cells}

NR8383 AM cells were cultured in Dulbecco's Modified Eagle Medium (DMEM) supplemented with $10 \%$ fetal bovine serum in 6-well plates at $37{ }^{\circ} \mathrm{C}$ and $5 \% \mathrm{CO}_{2}$. In the LPS group, cells were treated with LPS $(1 \mu \mathrm{g} / \mathrm{mL})$ for 24 hours and harvested. In the LPS + HRS group, 30 minutes after the introduction of LPS, $0.8 \mathrm{mM}$ HRS was added. In the HRS alone group, HRS was added at the same time as for the LPS + HRS group. The cell autophagy inhibitor 3-methyladenine (3-MA; $10 \mathrm{mM}$ ) was used to treat cells for 6 hours to inhibit autophagy. In the control group, an equivalent volume of NS was added.

\section{Histopathology}

Lungs were harvested at different time points following HRS treatment (4, 8, 12, and 24 hours) to determine histological changes. Lungs were fixed in $10 \%$ formalin for 24 hours at $4{ }^{\circ} \mathrm{C}$ and embedded in paraffin. Lung sections $(5 \mu \mathrm{m})$ were stained with hematoxylin-eosin (HE). Histopathologic scores were allocated by a pathologist blinded to the treatments, as previously described (8). Briefly, each of the following 5 features including edema, congestion, intra-alveolar hemorrhage or debris, neutrophil infiltration, and cellular hyperplasia were graded as absent, mild, moderate, or severe, using a score of 0 to 3 , giving a total score of 0 to 15 .

\section{Transmission electron microscopy (TEM)}

Cells were digested and centrifuged at $800 \mathrm{rpm}$ for 5 minutes. The cell pellet was collected, fixed with $2.5 \%$ glutaraldehyde overnight, followed by fixation with $1 \%$ osmium acid solution for 2 hours. Samples were dehydrated, infiltrated with dimethyl sulfoxide, embedded, ultrathin sectioned, stained with $2 \%$ tannic acid, and examined using the Hitachi Model H-7650 transmission electron microscope.

\section{Immunofluorescence}

Tissue slices were dewaxed and placed in citric acid buffer. The samples were boiled in a microwave 3 times for 5 minutes for antigen repair and then washed 3 times with phosphate buffered saline (PBS), each for 3 minutes, at room temperature. Tissue sections were incubated with primary antibodies against F4/80 (1:200, ab6640, Abcam, Cambridge, UK), inducible nitric oxide synthase (iNOS; 1:200, ab15323, Abcam, Cambridge, UK), arginase 1 (Arg-1; 1:50, \#93668, Cell Signaling Technology/CST, MA, USA), or light chain 3B (LC3B; 1:200, \#3868, CST, MA, USA) at room temperature for 1 hour. After washing 3 times with PBS, samples were incubated with goat anti-rat $\operatorname{IgG}$ H\&L (Cy3) (1:100, ab6953, Abcam, Cambridge, UK) or goat anti-rabbit IgG H\&L (FITC) (1:100, ab6717, Abcam, Cambridge, UK) secondary antibodies, at room temperature for 1 hour. Tissues were counterstained with 4',6-diamidino2-phenylindole (DAPI) for 20 minutes at room temperature, protected from light. An anti-fluorescence quencher was used for sealing. Protein expression was visualized using fluorescence microscopy (Olympus Corporation, Japan). Positive cells in the photomicrographs were counted using Image J software (National Institutes of Health, USA). Five random fields of view were examined ( $\times 200$ magnification).

\section{TUNEL staining}

Lung sections were stained using the TUNEL kit (No. 11684817910, Roche, USA) according to the manufacturer's instructions. Briefly, paraformaldehyde-fixed lung tissue were routinely dehydrated, embedded, and sectioned. According to the number of slides and the size of the tissues, the appropriate amount of reagent 1 (TdT) and reagent 2 (dUTP) in the TUNEL kit were mixed in a 1:9 ratio and added to the tissues. Sections were placed in a wet box in a $37^{\circ} \mathrm{C}$ incubator for 2 hours and blocked with $3 \%$ bovine serum albumin (BSA) at room temperature for 30 minutes. Biotinylated nucleotides and terminal deoxynucleotidyl transferase were added to the sections (1:500 dilution) and incubated at $4{ }^{\circ} \mathrm{C}$ overnight. After washing with PBS, sections were incubated with horseradish peroxidase (HRP)-labeled streptavidin in the dark for 50 minutes. Sections were counterstained with DAPI for 10 minutes at 
room temperature in the dark. Slides were sealed with an anti-fluorescence quenching agent. Five random fields of view per slide (×200 magnification) were visualized using fluorescence microscopy (Olympus Corporation, Japan). Positive cells in the photographs were counted using the Image J software (National Institutes of Health, USA).

\section{Flow cytometry}

NR8383 cells were trypsinized, washed, and resuspended in PBS $\left(1 \times 10^{6}\right.$ cells $)$. To investigate apoptosis, $5 \mu \mathrm{L}$ Annexin V (556547, BD Pharmingen, San Diego, CA, USA) and $5 \mu \mathrm{L}$ propidium iodide (PI; 556547, BD Pharmingen, San Diego, CA, USA) were added to the cells and incubated for 30 minutes at room temperature, protected from light. Cells were then washed with PBS and resuspended in $300 \mu \mathrm{L}$ of PBS. The apoptosis ratio was calculated using Flow Jo software (BD Biosciences, USA).

For detection of cell polarization, $1 \times 10^{5} \mathrm{NR} 8383$ cells were resuspended in $100 \mu \mathrm{L}$ PBS, and $1 \mu \mathrm{L}$ APC-labeled iNOS antibody (eBioscience, USA) and $1 \mu \mathrm{L}$ PE-labeled Arg1 antibody (eBioscience, USA) were added. Cells were then incubated for 30 minutes on ice, in the dark. Thereafter, cells were washed and resuspended in $300 \mu \mathrm{L}$ of PBS. iNOS and Arg1 were measured by flow cytometry.

\section{Enzyme-linked immunosorbent assay (ELISA)}

The cytokines interleukin (IL)-1 $\beta$, IL-6, and tumor necrosis factor (TNF)- $\alpha$ were detected in lung tissue using ELISA kits (KeyGEN, Nanjing, China) according to the manufacturer's protocols. All experiments were performed in triplicates.

\section{Western blot analysis}

Cells or tissues were homogenized or lysed, respectively, with RIPA Lysis and Extraction Buffer (89900, Thermo Fisher Scientific, Inc., Waltham, MA, USA) containing protease inhibitors. Protein concentration was determined using the BCA protein assay kit (Thermo Fisher Scientific, Inc.). Protein samples (20 $\mu \mathrm{g}$ per well) were separated by SDS-PAGE (sodium dodecyl sulphate-polyacrylamide gel electrophoresis) and transferred to PVDF (polyvinylidene difluoride) membranes. Blots were incubated with iNOS (1:1,000, ab15323, Abcam, Cambridge, UK), Arg-1 (1:1,000, \#93668, CST, MA, USA), Bax (1:1,000, ab32503, Abcam, Cambridge, UK), Bcl-2 (1:1,000, ab59348, Abcam,
Cambridge, UK), cleaved caspase-3 (1:1,000, \#9664, CST, MA, USA), LC3A/B (1:1,000, \#4108, CST, MA, USA), or p62 (1:1,000, ab109012, Abcam, Cambridge, UK) primary antibodies, at $4{ }^{\circ} \mathrm{C}$ overnight. After washing several times with PBS, the membranes were incubated with horseradish peroxidase (HRP)-labeled secondary antibody (1:2,000, \#7074, CST, MA, USA) for 2 hours at room temperature. Protein bands were visualized using an ECL chemiluminescence detection kit (cat. no. 34096, Thermo Scientific, USA) and a gel imaging system (Gel Doc $\left.^{\mathrm{TM}} \mathrm{XR} ; 170-8170\right)$. Band intensity was quantified using Image J software (National Institutes of Health, Bethesda, MD, USA).

\section{Statistical analysis}

All experiments were repeated at least 3 times. All outcome measures were assessed by a blinded observer. and data analyses were performed using SPSS 20.0 (SPSS Inc., Chicago, IL, USA). Results are expressed as mean \pm standard deviation (SD). All data were analyzed by oneway analysis of variance (ANOVA) with Tukey's multiple comparison post hoc test. Histological scores were analyzed by the Kruskal-Wallis test. A P value $<0.05$ was considered statistically significant.

\section{Results}

\section{HRS attenuated LPS-induced ALI in rats}

The effects of HRS on LPS-induced lung injury in rats were examined at different time points (4, 8, 12, and 24 hours; Figure $1 A$ ). Based on histopathological scoring, lung injury peaked at 24 hours post-LPS challenge $(\mathrm{P}<0.001$; Figure $1 B)$, consistent with previous results (8). Thus, 24 hours was selected as the observation time point for subsequent experiments. HE staining of lung tissue showed that LPSinduced lung injury was characterized by destruction of alveolar structure, inflammatory cell infiltration into the pulmonary interstitium and alveoli, and intra-alveolar hemorrhage (Figure 1A). HRS attenuated all signs of LPSinduced lung injury. The mean histological score was higher in the LPS group compared to the control or the HRS group, and the effects of LPS were significantly reduced by HRS treatment (LPS + HRS; $\mathrm{P}<0.01$; Figure $1 C$ ). Additionally, levels of IL-6, IL-1 $\beta$, and TNF- $\alpha$ in lung tissues was significantly increased in rats with LPS-induced lung injury. However, HRS attenuated the increase in 


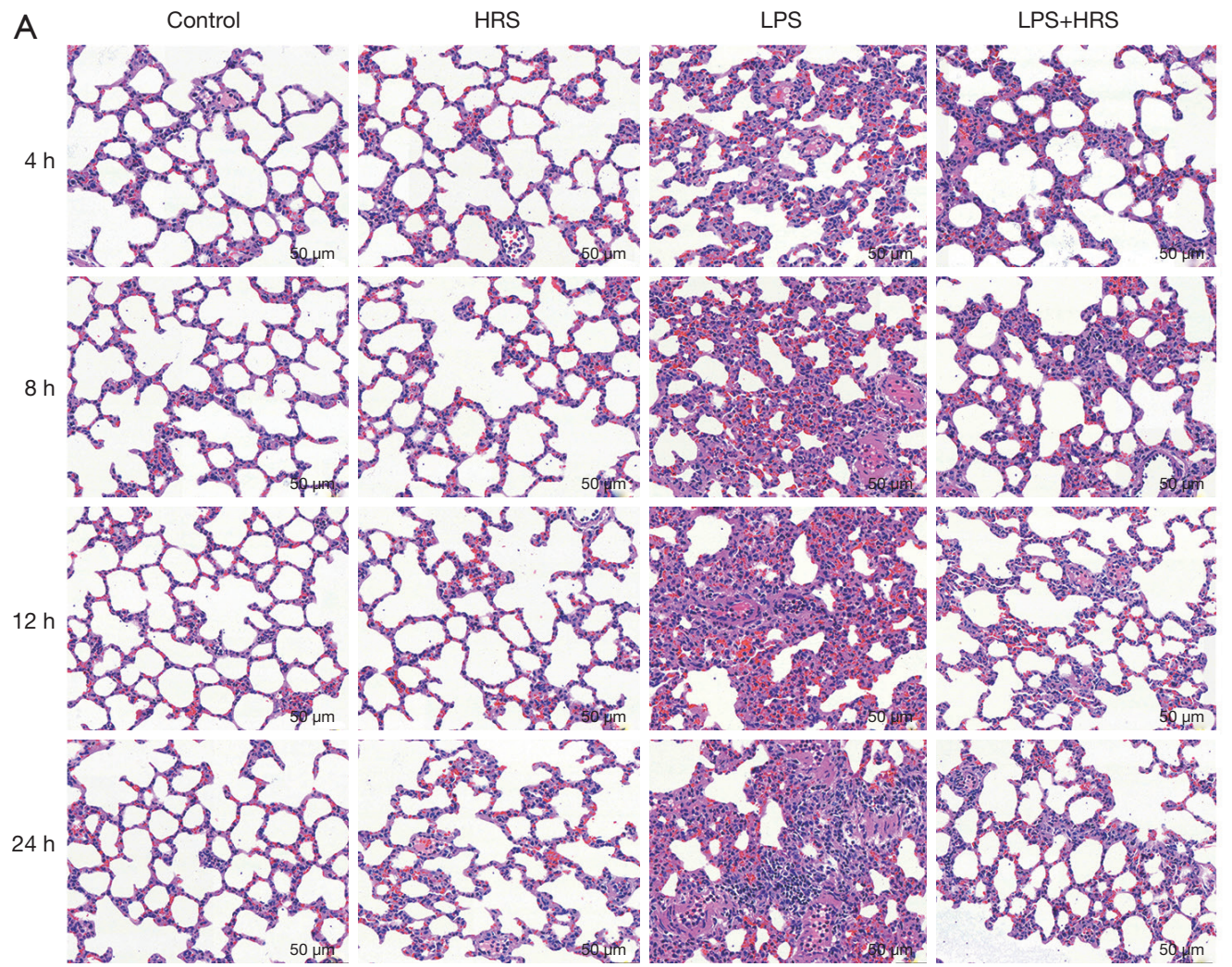

B

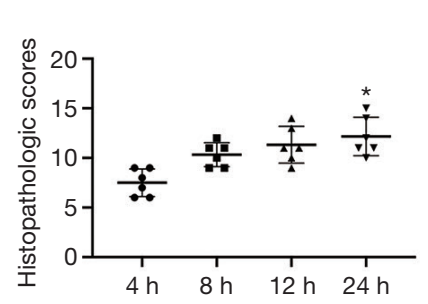

C

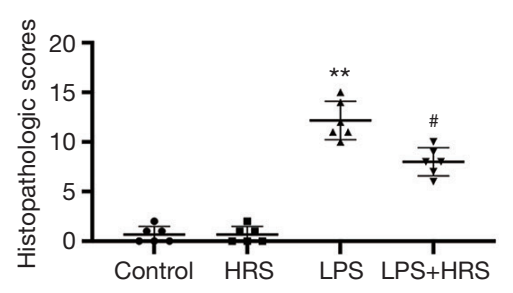

D

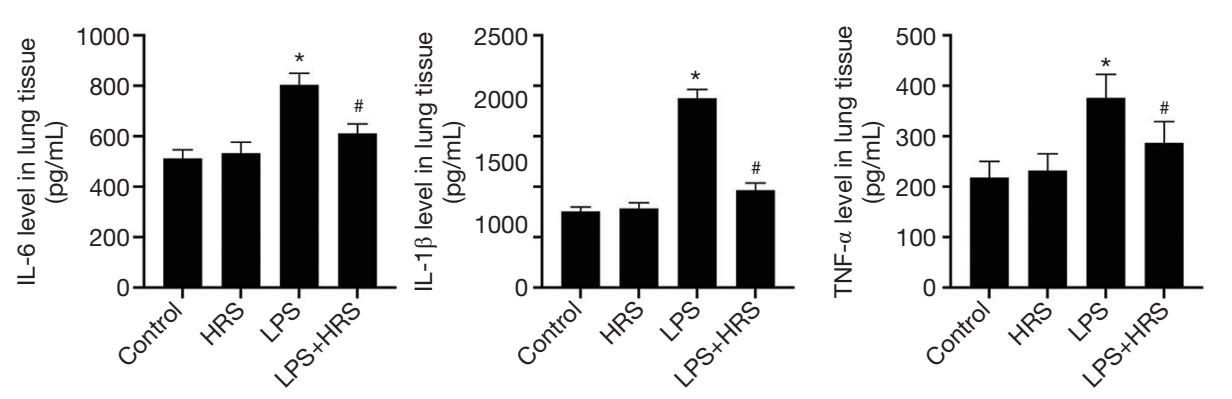

Figure 1 HRS ameliorated the histopathological changes in the lungs of LPS-challenged rats. (A) Rat lungs (n=6 per group) were collected for HE staining 4, 8, 12, or 24 hours after the indicated treatments. Scale bar $=50 \mu \mathrm{m}$. (B) Histological scores in rat lungs after LPS treatment at different time points. (C) Histological scores in rat lungs at 24 hours for the indicated treatment groups. (D) Levels of IL6 , IL-1 $\beta$, and TNF- $\alpha$ in lung tissues. Data are the mean \pm SD of three independent experiments. ${ }^{*} \mathrm{P}<0.001$ compared to other time points; ${ }^{* *} \mathrm{P}<0.01$ compared to the control group; ${ }^{~} \mathrm{P}<0.01$ compared to the LPS group; "⿴囗十, $, \mathbf{\Lambda}, \boldsymbol{\nabla}$ " represent the histopathologic scores of lung tissue in different rats. HRS, hydrogen-rich saline; LPS, lipopolysaccharide; HE, hematoxylin eosin; IL, interleukin; TNF, tumor necrosis factor; SD, standard deviation. 
cytokine levels $(\mathrm{P}<0.05$; Figure $1 D)$. In summary, HRS significantly alleviated lung injury induced by LPS in Sprague Dawley rats.

\section{HRS decreased polarization from M2 to M1 and attenuated AM apoptosis in septic rats}

Since polarization and apoptosis of AMs are key factors in the pathogenesis of ALI $(2,11)$, the effects of LPS and HRS on AMs were examined by immunofluorescence staining. More M1 AMs (F4/80 and iNOS positive cells) were detected in the lungs of LPS-challenged rats than in control animals. Treatment with HRS reduced this cell population in LPS-challenged rats (Figure $2 A$ ). By contrast, the numbers of M2 macrophages (F4/80 and Arg-1 positive cells) decreased in LPS-challenged rats but increased after HRS treatment (Figure 2B). Consistent with the histological results, rats in the LPS group had higher numbers of M1 and lower numbers of M2 macrophages compared to animals in the control group and the HRS group $(\mathrm{P}<0.01 ;$ Figure $2 C)$. When LPS-challenged animals were administered HRS, the number of M1 macrophages decreased, while the number of M2 macrophages increased compared to untreated LPS-challenged rats $(\mathrm{P}<0.01$; Figure 2C). Furthermore, LPS-challenged rat lungs had more apoptotic AMs (F4/80 and TUNEL positive cells) compared to control rats (Figure $3 A$ ), and this was reduced after treatment with HRS $(\mathrm{P}<0.01 ;$ Figure $3 B)$. These results suggested that HRS decreased the polarization of AMs from M2 to M1 and attenuated apoptosis in this cell population during sepsis.

\section{HRS suppressed LPS-induced autophagy in AMs of septic rats}

As autophagy is involved in both the regulation of AM polarization and apoptosis, the effects of LPS and HRS on autophagy of AMs in septic rats were investigated. Lung tissue from rats challenged with LPS showed significantly higher levels of autophagy (F4/80 and LC3B positive cells) compared to lung tissue from control rats (Figure $4 A$ ). These heightened levels of autophagy were suppressed after administration of HRS (Figure 4A). The mean levels of autophagy in the LPS + HRS group and the HRS group were significantly lower than in the LPS group $(\mathrm{P}<0.01$; Figure $4 B)$. However, no significant differences were found between the control and HRS groups $(\mathrm{P}>0.05$; Figure $4 B)$. These findings suggested that HRS can suppress autophagy in AMs challenged by LPS, but it does not affect normal cells.

\section{HRS decreased M2 to M1 polarization, attenuated apoptosis, and suppressed autophagy in LPS-challenged NR8383 rat AM cells in vitro}

To confirm the in vivo results, the rat AM cell line NR8383 was used for in vitro experiments. The markers of macrophage polarization, iNOS (M1) and Arg-1 (M2), were quantified by Western blot in $(\mathrm{P}<0.05$, Figure $5 A)$ and immunofluorescence $(\mathrm{P}<0.05$, Figure $5 B)$. These results showed that LPS increased the expression of iNOS $(\mathrm{P}<0.05)$ and decreased Arg-1 protein expression $(\mathrm{P}<0.05)$. These effects were reversed by HRS $(\mathrm{P}<0.05)$. In addition, flow cytometry demonstrated that the M2/M1 ratio of the LPS group decreased significantly compared to both the control and HRS groups $(\mathrm{P}<0.05$; Figure $5 C)$. This ratio increased in LPS-treated cells in the presence of HRS $(\mathrm{P}<0.05$; Figure $5 C)$. Western blotting and Annexin V-PI were used to assess apoptosis in NR8383 cells 24 hours after treatment. The $\mathrm{Bax} / \mathrm{Bcl}-2$ ratio increased significantly in cells treated with LPS compared to control cells $(\mathrm{P}<0.05$; Figure 5D). Furthermore, the levels of cleaved caspase- 3 were also elevated in the LPS group compared to the control group $(\mathrm{P}<0.05$; Figure $5 D)$, suggesting increased apoptosis. These effects were reversed by HRS treatment. Consistent with the Western blot results, Annexin V-PI showed that the proportion of apoptotic cells in the LPS group was increased compared to the control group and the HRS group $(\mathrm{P}<0.05$; Figure $5 E)$. Again, HRS decreased the apoptosis ratio significantly in LPS-treated cells $(\mathrm{P}<0.05$; Figure 5E).

The expression levels of proteins related to autophagy, including LC3-I, LC3-II, and p62, were assessed by Western blot. The LC3II/I ratio, representing autophagy, was significantly higher in the LPS group compared to the control group and the HRS group $(\mathrm{P}<0.05$; Figure $6 A)$. HRS markedly decreased this ratio in LPS-treated cells $(\mathrm{P}<0.05$; Figure $6 A$ ). Furthermore, the mean level of p62 decreased after LPS administration, and this was reversed by HRS $(\mathrm{P}<0.05$; Figure $6 A)$. Moreover, the immunofluorescence results showed the expression of LC $3 \mathrm{~B}$ was upregulated in the LPS group compared to that in the control group; HRS markedly reduced the LC 3B expression in LPStreated cells $(\mathrm{P}<0.05$; Figure $6 B)$. In addition, TEM results showed more autophagosomes (arrows), which contain integrated cytoplasmic components with double-membrane 

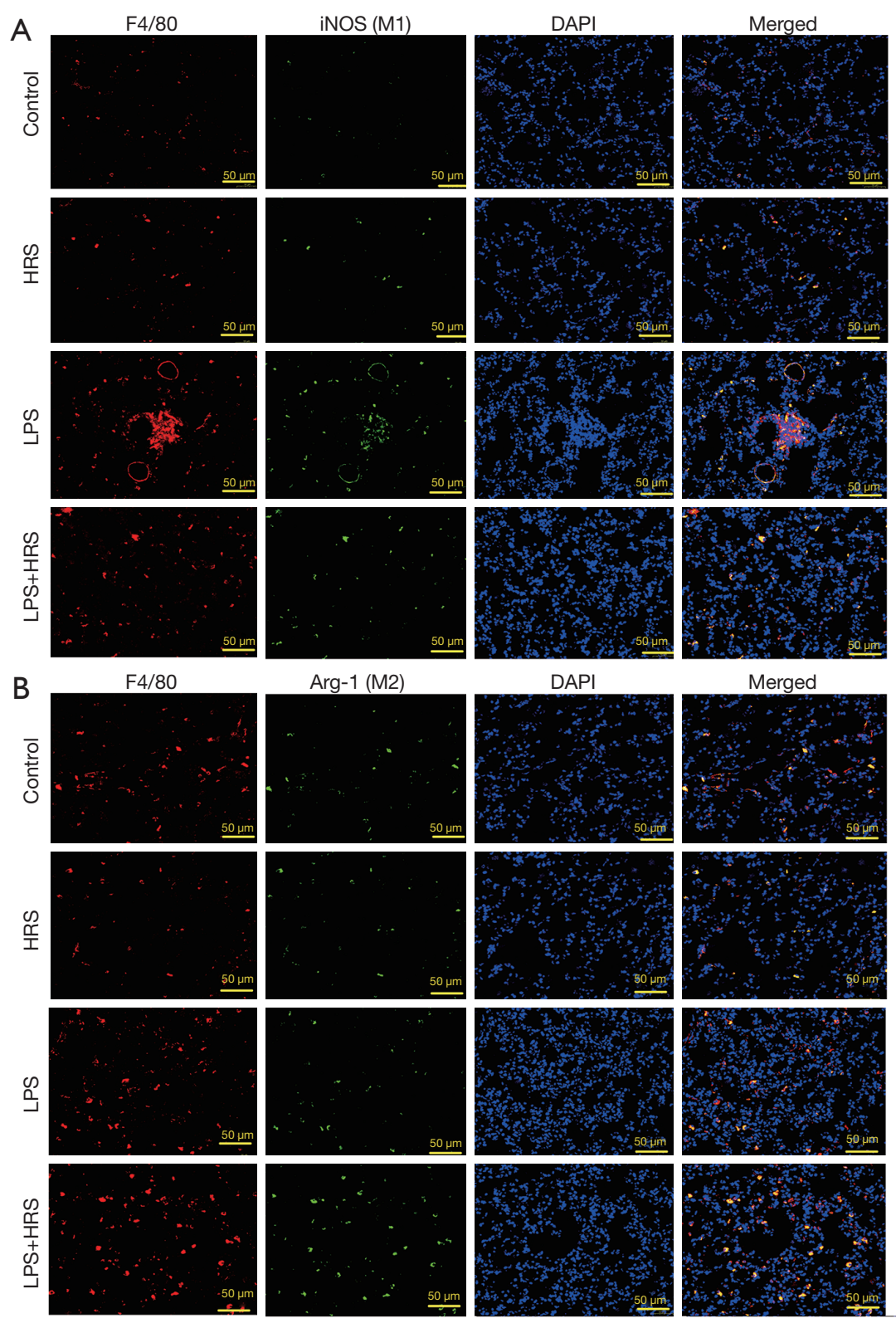

C

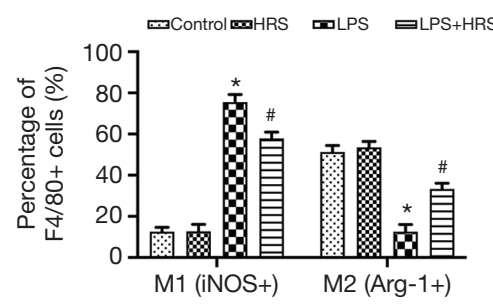

Figure 2 HRS treatment decreased M1 and increased M2 phenotypes of AMs in LPS-challenged rats. Immunofluorescence staining of lung tissue to identify (A) M1 macrophages (F4/80 and iNOS positive cells) and (B) M2 macrophages (F4/80 and Arg-1 positive cells) 24 hours after the indicated treatments. F4/80-positive cells (red); iNOS or Arg-1 positive cells (green). Nuclei were stained with DAPI (blue). Scale bar $=50 \mu \mathrm{m}$. (C) Quantification of M1 and M2 macrophages in lung tissue. Data are the mean \pm SD of three independent experiments. ${ }^{*} \mathrm{P}<0.01$ compared to the control group; ${ }^{\#} \mathrm{P}<0.01$ compared to the LPS group. HRS, hydrogen-rich saline; LPS, lipopolysaccharide; AMs, alveolar macrophages; iNOS, inducible nitric oxide synthase; DAPI, 4',6-diamidino-2-phenylindole; SD, standard deviation. 

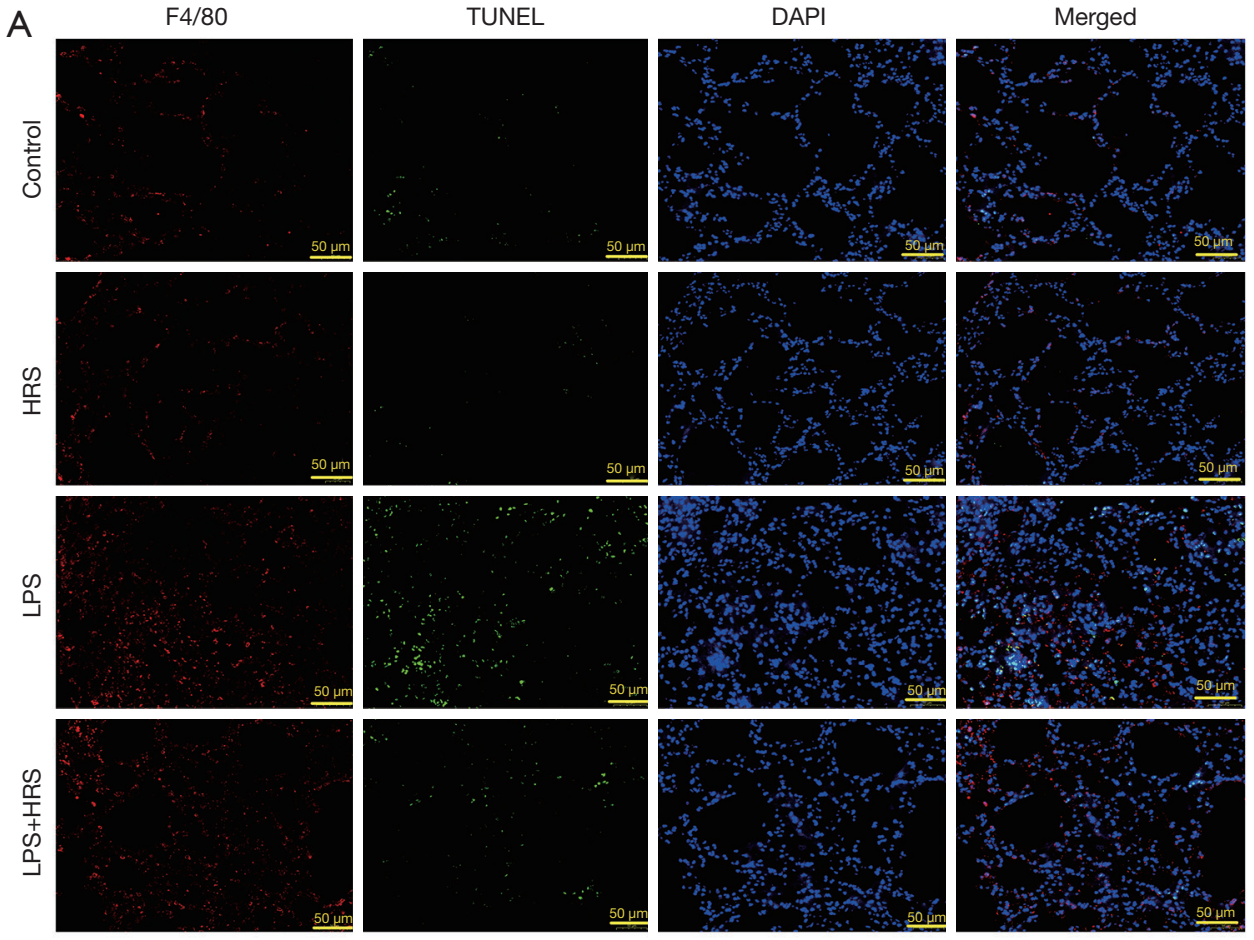

B

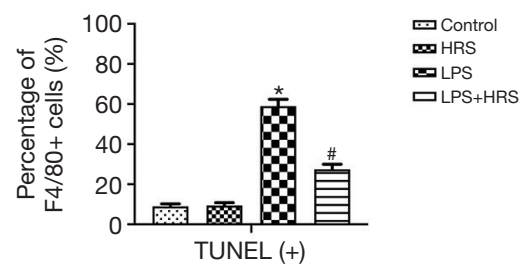

Figure 3 HRS treatment inhibited AM apoptosis in LPS-challenged rats. (A) F4/80 (red) and TUNEL (green) positive cells represent apoptotic AMs in lung tissue 24 hours after administration of the indicated treatments. (B) Quantification of macrophage apoptosis in lung tissue. Scale bar $=50 \mu \mathrm{m}$. Data are shown as mean $\pm \mathrm{SD}$ of three independent experiments. ${ }^{*} \mathrm{P}<0.01$ compared to the control group; ${ }^{\#} \mathrm{P}<0.01$ compared to the LPS group. HRS, hydrogen-rich saline; LPS, lipopolysaccharide; AM, alveolar macrophage; DAPI, 4',6-diamidino-2phenylindole; SD, standard deviation.

structures, in LPS-challenged cells compared to control cells (Figure 6C). Fewer autophagosomes were observed in LPSchallenged cells in the presence of HRS (Figure 6C). Taken together, these findings demonstrated that HRS decreased the polarization of AMs from M2 to M1, attenuated apoptosis, and suppressed autophagy in LPS-challenged NR8383 cells.

\section{HRS regulated polarization and inbibited apoptosis through the suppression of autophagy in LPS-challenged NR8383 cells}

To determine whether HRS could regulate polarization and apoptosis through autophagy, cells were treated with the autophagy inhibitor 3-MA. As shown in Figure $7 A$, 3-MA significantly decreased the LC3II/I ratio and increased p62 expression in NR8383 cells, indicating lower autophagy levels following 3-MA treatment compared to cells treated with LPS $(\mathrm{P}<0.05)$. In LPS-challenged cells, addition of 3-MA significantly decreased iNOS expression $(\mathrm{P}<0.05$; Figure $7 A)$ and the ratio of $\mathrm{Bax} / \mathrm{Bcl}$ $2(\mathrm{P}<0.05$; Figure $7 A)$, as well as the expression of cleaved caspase-3 $(\mathrm{P}<0.01$; Figure $7 A)$ compared to untreated LPS-challenged cells. In contrast, expression of Arg-1 $(\mathrm{P}<0.05$; Figure $7 A)$ increased markedly in the LPS + 3-MA group compared to the LPS alone group. Moreover, the immunofluorescence results showed the iNOS, Arg-1 

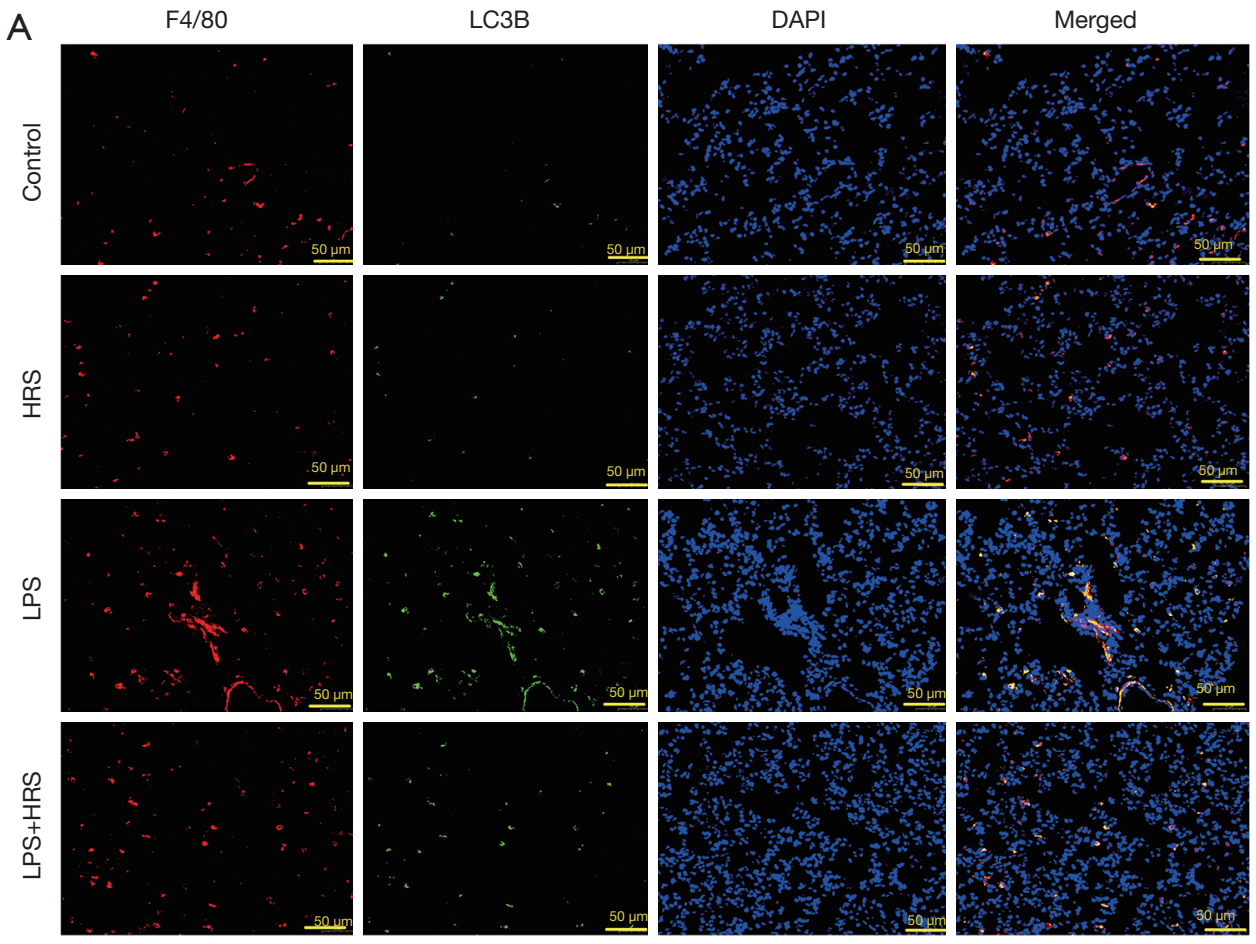

B

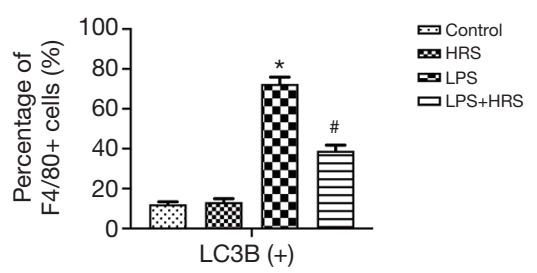

Figure 4 HRS suppressed autophagy in AMs in LPS-challenged rats. (A) F4/80 (red) and LC3B (green) positive cells represent macrophages undergoing autophagy in lung tissue 24 hours after administration of the indicated treatments. (B) Quantification of macrophage autophagy in lung tissue. Scale bar $=50 \mu \mathrm{m}$. Data are shown as mean $\pm \mathrm{SD}$ of three independent experiments. ${ }^{*} \mathrm{P}<0.01$ compared to the control group; ${ }^{\#} \mathrm{P}<0.01$ compared to the LPS group. HRS, hydrogen-rich saline; LPS, lipopolysaccharide; AM, alveolar macrophage; SD, standard deviation; DAPI, 4',6-diamidino-2-phenylindole.

positive cells and the expression of LC3B in NR8383 cells, the results is same with the results of western blot $(\mathrm{P}<0.05$; Figure $7 B$ and $C$ ). Consistent with the western blotting results, flow cytometry showed a higher $M 2 / \mathrm{M} 1$ ratio $(\mathrm{P}<0.05$; Figure $7 D)$ and lower apoptosis ratio $(\mathrm{P}<0.05$; Figure $7 E)$ in the LPS + 3-MA group compared to the LPS alone group. Additionally, no significant differences were observed between the LPS + 3-MA, LPS + HRS, and LPS + HRS + 3-MA groups ( $>0.05$; Figure 7), suggesting that HRS and 3-MA had similar effects on AM cells. In summary, this data demonstrated that HRS regulated AM polarization and inhibited apoptosis through suppression of autophagy in LPS-challenged NR8383 cells.

\section{Discussion}

In recent years, a number of publications have focused on the protective effect of HRS against ALI in sepsis (5-8). Lung tissue contains high numbers of alveolar epithelial cells, vascular endothelial cells, and interstitial AMs. In previous studies, HRS has been found to reduce apoptosis (7), downregulate aquaporin expression in alveolar epithelial cells (5), and improve vascular endothelial cell dysfunction in septic rat lungs (6). As resident pulmonary immune cells, AMs are involved in the recruitment of neutrophils in the early stages of inflammation, in the release of inflammatory cytokines and chemokines, and in tissue repair in the later stages of inflammation $(2,10,11)$. However, the effects of HRS on 

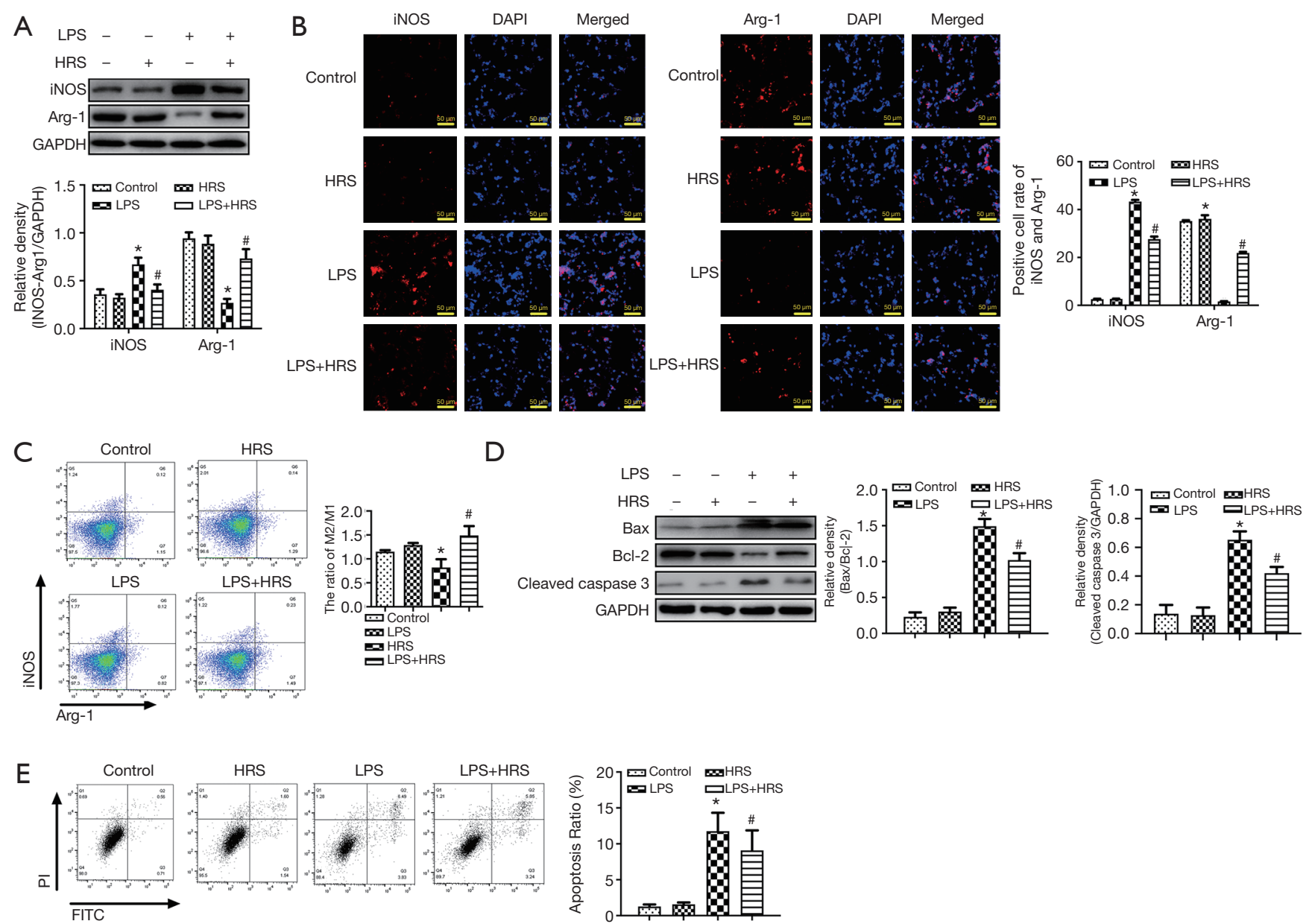

Figure 5 HRS treatment increased the M2 phenotype and decreased the M1 phenotype and apoptosis in LPS-challenged NR8383 cells. (A) Western blot and densitometric quantification of iNOS and Arg-1. (B) Immunofluorescence results of iNOS and Arg-1 in NR8383 and the positive cell rate analysis. (C) The phenotypic ratio of M2 to M1. (D) The western blot results and their analysis of Bax, Bcl-2, and cleaved caspase-3. GAPDH was used as an internal control. (E) Flow cytometry was used to determine the apoptotic ratio in NR8383 cells. Data are shown as mean $\pm \mathrm{SD}$ of three independent experiments. ${ }^{*} \mathrm{P}<0.05$ compared to the control group; ${ }^{\#} \mathrm{P}<0.05$ compared to the LPS group. HRS, hydrogen-rich saline; LPS, lipopolysaccharide; AMs, alveolar macrophages; iNOS, inducible nitric oxide synthase; Arg-1, arginase 1; GAPDH, glyceraldehyde 3-phosphate dehydrogenase; PI, propidium iodide; FITC, fluorescein isothiocyanate; SD, standard deviation.

macrophages during sepsis have remained unexplored. Phenotypic changes and apoptosis of macrophages play an important role in the pathogenesis of sepsis and ALI or ARDS $(2,10,11)$. To our knowledge, this present study is the first to examine the effects of HRS on the function of AMs during sepsis. Administration of LPS resulted in severe lung injury and increased M1 polarization and apoptosis in AMs. However, HRS treatment reduced the M1 phenotype, increased the M2 phenotype, and reduced apoptosis in LPS-challenged AMs. Excessive activation of M1 is the main source of proinflammatory factors in uncontrolled inflammation, and apoptosis of macrophages is an important cause of immune suppression $(2,10,11)$. Therefore, the data from this study suggested that HRS may attenuate LPSinduced ALI through the regulation of polarization and reduction of apoptosis in AMs.

As an important protective and renewal mechanism, autophagy is increasingly recognized as a vital factor in inflammation and immunization (14,20). As summarized in our previous review, macrophage autophagy is a common regulatory mechanism for self-polarization and apoptosis in multiple diseases (21). Furthermore, 

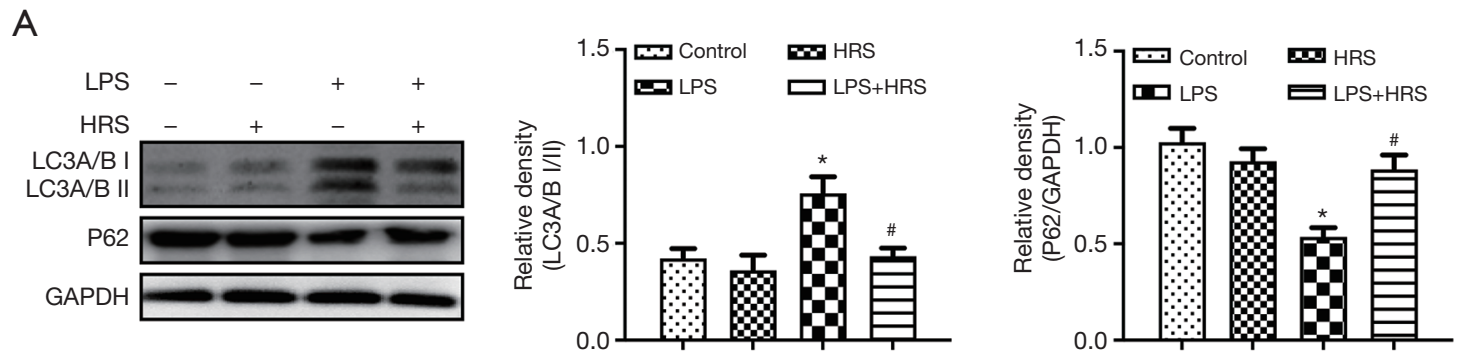

B

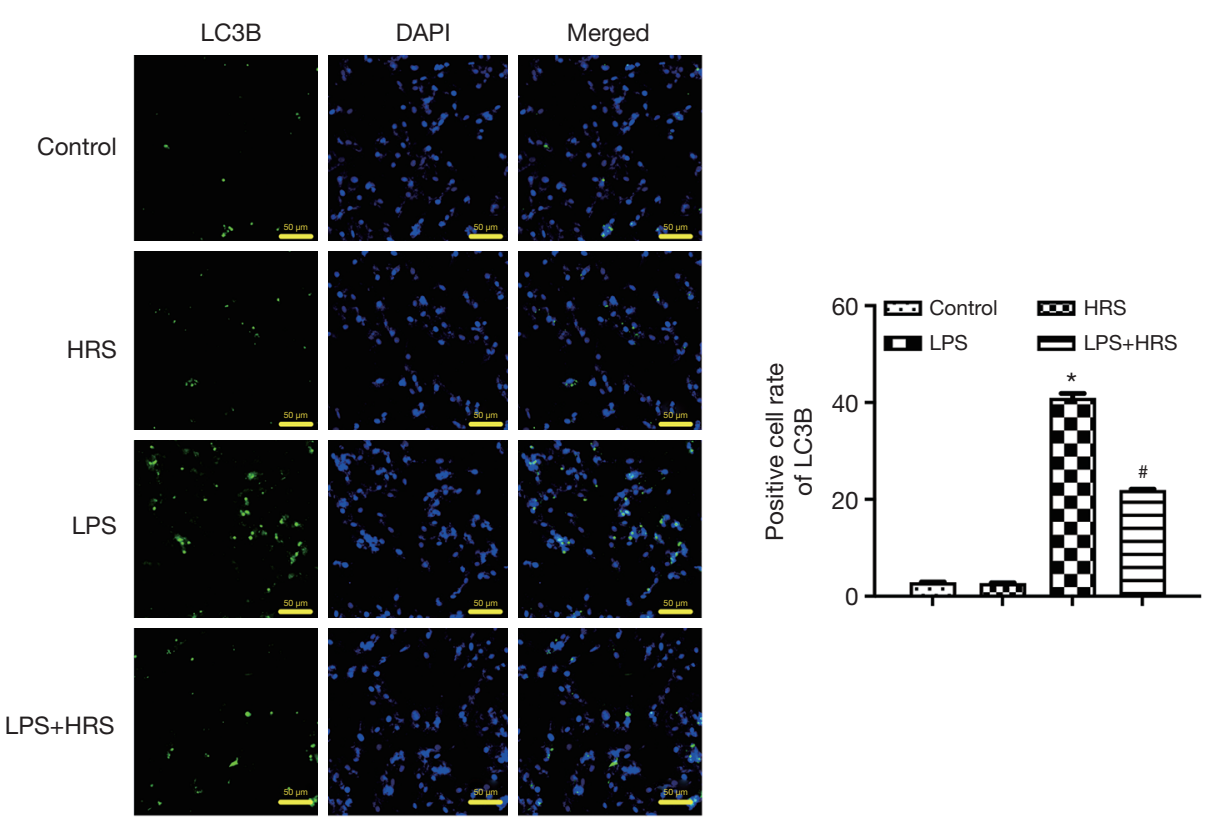

C
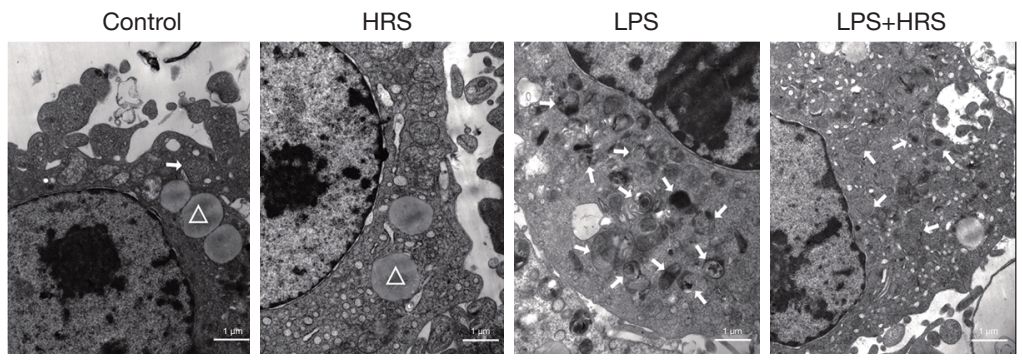

Figure 6 HRS treatment suppressed autophagy in LPS-challenged NR8383 cells. (A) Western blot and densitometric quantification of LC3A/B I, LC3A/B II, and p62. (B) Immunofluorescence results and quantification of LC3B. Scale bar $=50 \mu \mathrm{m}$. (C) Autophagosomes in AMs were observed using TEM (white arrows, $\times 20,000$ magnification). The triangles $(\Delta)$ indicate lysosomes in AMs. Scale bar $=1 \mu \mathrm{m}$. Data are shown as mean $\pm \mathrm{SD}$ of three independent experiments. ${ }^{*} \mathrm{P}<0.05$ compared to the control group; ${ }^{\#} \mathrm{P}<0.05$ compared to the LPS group. HRS, hydrogen-rich saline; LPS, lipopolysaccharide; AM, alveolar macrophage; TEM, transmission electron microscopy; SD, standard deviation.

studies have demonstrated that molecular hydrogen plays a protective role by modulating autophagy in multiple diseases, including sepsis $(7,22)$, ischemia-reperfusion injury (23), organ transplantation (24), and pathological neuralgia (25). However, the specific regulatory effects of HRS on autophagy of macrophages during sepsis has not been previously explored. The experiments in this current report suggested that HRS has an inhibitory effect on LPS- 
A

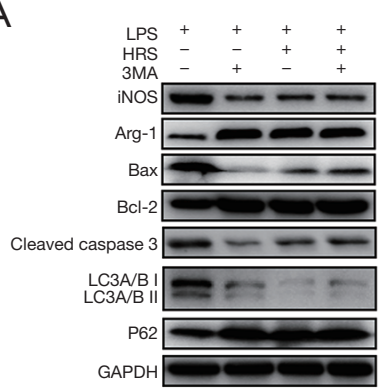

B
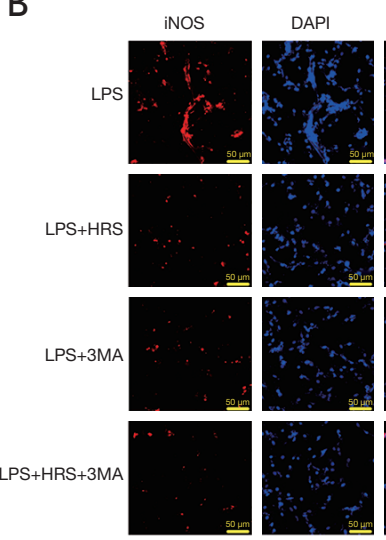
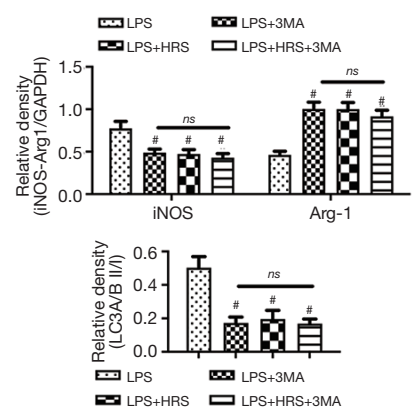
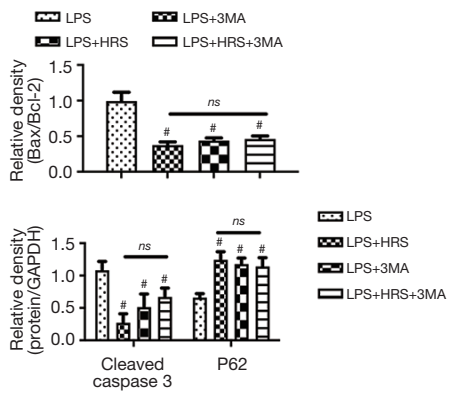

C
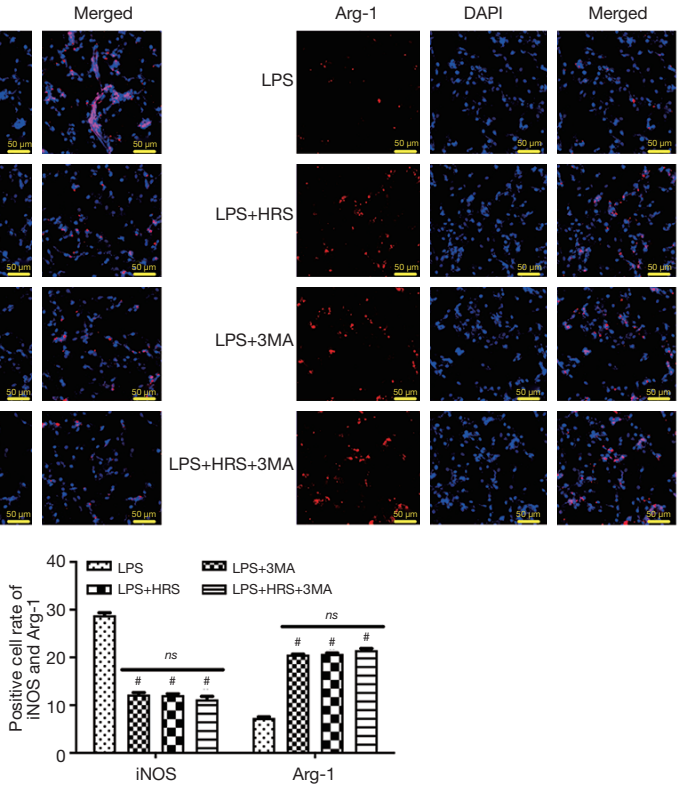

D
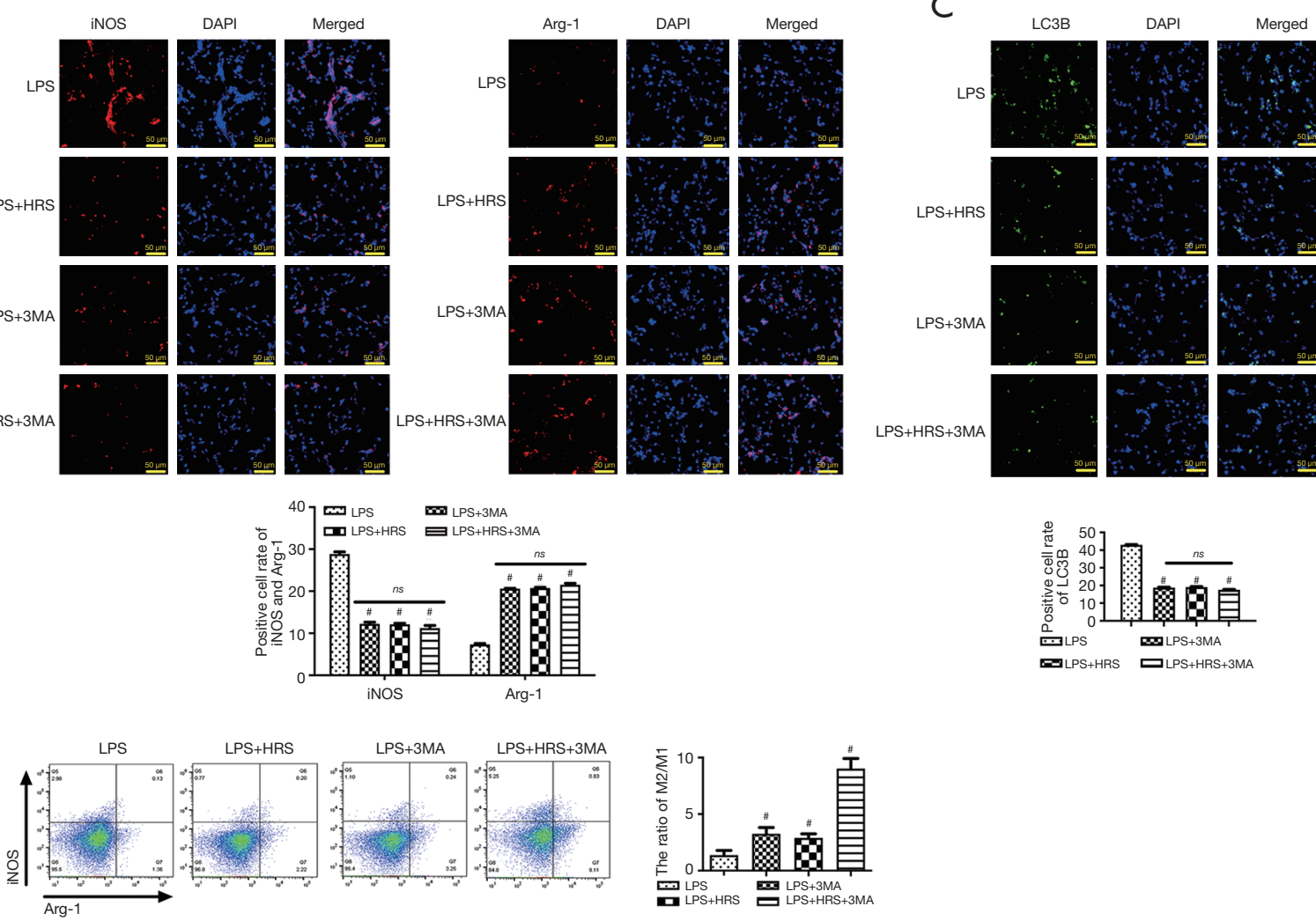

$E$
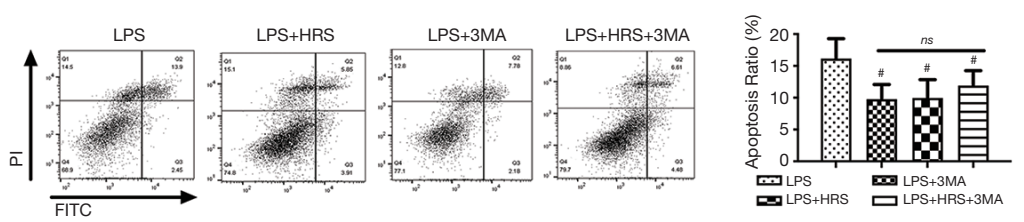

Figure 7 HRS treatment modulated apoptosis and polarization through suppression of autophagy in LPS-challenged NR8383 cells. (A) Western blot and its relative density analysis of iNOS, Arg-1, Bax, Bcl-2, cleaved caspase-3, LC3A/B I, LC3A/B II, and p62 expression in NR8383 cells. (B) Immunofluorescence result of iNOS and Arg-1 in NR8383 and the positive cell rate analysis. (C) Immunofluorescence result of LC3B in NR8383 and positive cell rate. (D,E) Flow cytometry was used to determine the M2/M1 ratio and apoptotic ratio in NR8383 cells. Data are shown as mean \pm SD of three independent experiments. ${ }^{~} \mathrm{P}<0.05$ compared to LPS group. ns, not significant; HRS, hydrogen-rich saline; LPS, lipopolysaccharide; 3MA, 3-methyladenine; AMs, alveolar macrophages; iNOS, inducible nitric oxide synthase; Arg-1, arginase 1; GAPDH, glyceraldehyde 3-phosphate dehydrogenase; PI, propidium iodide; FITC, fluorescein isothiocyanate; SD, standard deviation. 
induced autophagy of AMs.

Autophagy is a complex and dynamic process, which plays different roles under different pathological conditions. The effect of autophagy on macrophage polarization in cancer remains controversial (26-28), and its effect in sepsis has not been previously investigated. This study found that LPS induced autophagy of AMs, promoting their transformation from M2 to M1. There is still some debate regarding the regulatory effects of autophagy on macrophage apoptosis $(17,29)$. The present study demonstrated that LPS-induced AM autophagy which enhanced apoptosis, and this was consistent with the findings of $\mathrm{Hu}$ et al. (17) but contrary to those reported by Zhang et al. (29). These differences may be explained by different levels of macrophage autophagy caused by different LPS concentrations and treatment durations. Autophagy is a double-edged sword. Moderate autophagy protects cells from stress-induced injury, while excessive autophagy tends to cause cell death (17,30-32). The level of autophagy depends on multiple factors, including cell type, pathological state, and stimulus intensity $(30,31)$. In these current experiments and those performed by $\mathrm{Hu} e t$ al., autophagy was induced by a severe systemic inflammatory response. In contrast, Zhang et al. (29) used a two-fold concentration of LPS and greater duration of exposure, which may have contributed to increased autophagy and, consequently, greater apoptosis.

Previous studies have shown that excessive pulmonary autophagy can aggravate LPS-induced ALI $(3,7)$. However, those latter studies examined overall autophagy in multiple types of lung tissue cells, whereas we focused exclusively on AMs, making our results more specific. According to the findings of Lo et al. (3), autophagy can protect lung tissue during the early stages of sepsis. However, in the later stages, lung injury is exacerbated due to the over-accumulation of autophagosomes with insufficient autolysosome fusion. These effects could explain the aggravated ALI observed in response to increased AM autophagy our experiments.

The present study demonstrated that LPS-induced autophagy of AMs increased both M1 polarization and apoptosis, which were reversed by 3-MA and HRS. Importantly, HRS and 3-MA exhibited similar effects on AMs in this study. Taken together, these results suggested that HRS reduces LPS-induced M1 polarization and apoptosis of AMs by suppressing autophagy. Despite the findings suggesting that HRS and 3-MA have similar regulatory effects on AMs, these agents may not necessarily play an equally protective role in septic lung injury. In previous studies, HRS has been demonstrated to exert biological effects, not only through the regulation of autophagy, but also through anti-oxidative (33), anti-inflammatory (7), antiapoptotic (33), and anti-shock effects (34) during sepsis. Therefore, it is likely that HRS has multiple benefits in the prevention of ALI caused by sepsis.

There are several limitations to the present study. First, autophagy may be part of a complex network regulating macrophage polarization and apoptosis. Additional studies are warranted to establish whether HRS also works through other mechanisms. Second, the exact mechanisms or signaling pathways by which HRS modulates AM autophagy require further investigation. In conclusion, these findings shed light on the role of HRS in AM polarization and apoptosis through the regulation of autophagy, suggesting a potential novel approach for the treatment of ALI caused by sepsis.

\section{Acknowledgments}

The authors thank BioMed Proofreading ${ }^{\circledR}$ LLC (Cleveland, Ohio, USA) for refining and polishing the manuscript.

Funding: This work was funded by the National Natural Science Foundation of China (Grant Number 81701951), the Liaoning Education Department (Grant Number LK201634), the 345 Talent Project and the Shenyang Municipal Science and Technology Commission (Grant Number 17-230-9-45).

\section{Footnote}

Reporting Checklist: The authors have completed the ARRIVE reporting checklist. Available at https://dx.doi. org/10.21037/atm-21-2489

Data Sharing Statement: Available at https://dx.doi. org/10.21037/atm-21-2489

Conflicts of Interest: All authors have completed the ICMJE uniform disclosure form (available at https://dx.doi. org/10.21037/atm-21-2489). The authors have no conflicts of interest to declare.

Ethical Statement: The authors are accountable for all aspects of the work in ensuring that questions related to the accuracy or integrity of any part of the work are appropriately investigated and resolved. All animal studies were performed with the approval of the China 


\section{Page 14 of 15}

Medical University Laboratory Animal Welfare and Ethics Committee (IACUC NO.2015048R) in accordance with the National Institutes of Health (Bethesda, MD, USA) guidelines for the care and use of laboratory animals.

Open Access Statement: This is an Open Access article distributed in accordance with the Creative Commons Attribution-NonCommercial-NoDerivs 4.0 International License (CC BY-NC-ND 4.0), which permits the noncommercial replication and distribution of the article with the strict proviso that no changes or edits are made and the original work is properly cited (including links to both the formal publication through the relevant DOI and the license). See: https://creativecommons.org/licenses/by-nc-nd/4.0/.

\section{References}

1. Stoller J, Halpin L, Weis M, et al. Epidemiology of severe sepsis: 2008-2012. J Crit Care 2016;31:58-62.

2. Huang X, Xiu H, Zhang S, et al. The Role of Macrophages in the Pathogenesis of ALI/ARDS. Mediators Inflamm 2018;2018:1264913.

3. Lo S, Yuan SS, Hsu C, et al. Lc3 over-expression improves survival and attenuates lung injury through increasing autophagosomal clearance in septic mice. Ann Surg 2013;257:352-63.

4. Ohsawa I, Ishikawa M, Takahashi K, et al. Hydrogen acts as a therapeutic antioxidant by selectively reducing cytotoxic oxygen radicals. Nat Med 2007;13:688-94.

5. Tao B, Liu L, Wang N, et al. Effects of hydrogen-rich saline on aquaporin 1, 5 in septic rat lungs. J Surg Res 2016;202:291-8.

6. Liu LD, Wu XY, Tao BD, et al. Protective effect and mechanism of hydrogen treatment on lung epithelial barrier dysfunction in rats with sepsis. Genet Mol Res 2016;15:gmr.15016050.

7. Zhang Y, Liu Y, Zhang J. Saturated hydrogen saline attenuates endotoxin-induced lung dysfunction. J Surg Res 2015;198:41-9.

8. Xie K, Yu Y, Huang Y, et al. Molecular hydrogen ameliorates lipopolysaccharide-induced acute lung injury in mice through reducing inflammation and apoptosis. Shock 2012;37:548-55.

9. Ichihara $M$, Sobue S, Ito $M$, et al. Beneficial biological effects and the underlying mechanisms of molecular hydrogen - comprehensive review of 321 original articles. Med Gas Res 2015;5:12.

10. Kumar V. Targeting macrophage immunometabolism:

\section{Qiu et al. HRS attenuates lung injury via reducing autophagy}

Dawn in the darkness of sepsis. Int Immunopharmacol 2018;58:173-85.

11. Johnston LK, Rims CR, Gill SE, et al. Pulmonary macrophage subpopulations in the induction and resolution of acute lung injury. Am J Respir Cell Mol Biol 2012;47:417-26.

12. Schultze JL, Schmidt SV. Molecular features of macrophage activation. Semin Immunol 2015;27:416-23.

13. Deretic V, Saitoh T, Akira S. Autophagy in infection, inflammation and immunity. Nat Rev Immunol 2013;13:722-37.

14. Matsuzawa-Ishimoto Y, Hwang S, Cadwell K. Autophagy and Inflammation. Annu Rev Immunol 2018;36:73-101.

15. Ip WKE, Hoshi N, Shouval DS, et al. Anti-inflammatory effect of IL-10 mediated by metabolic reprogramming of macrophages. Science 2017;356:513-9.

16. Chang CP, Su YC, Lee PH, et al. Targeting NFKB by autophagy to polarize hepatoma-associated macrophage differentiation. Autophagy 2013;9:619-21.

17. Hu R, Chen ZF, Yan J, et al. Complement C5a exacerbates acute lung injury induced through autophagymediated alveolar macrophage apoptosis. Cell Death Dis 2014;5:e1330.

18. Chen K, Wang N, Diao Y, et al. Hydrogen-Rich Saline Attenuates Brain Injury Induced by Cardiopulmonary Bypass and Inhibits Microvascular Endothelial Cell Apoptosis Via the PI3K/Akt/GSK3 $\beta$ Signaling Pathway in Rats. Cell Physiol Biochem 2017;43:1634-47.

19. Tao B, Liu L, Wang N, et al. Hydrogen-Rich Saline Attenuates Lipopolysaccharide-Induced Heart Dysfunction by Restoring Fatty Acid Oxidation in Rats by Mitigating C-Jun N-Terminal Kinase Activation. Shock 2015;44:593-600.

20. Deretic V, Kimura T, Timmins G, et al. Immunologic manifestations of autophagy. J Clin Invest 2015;125:75-84.

21. Qiu P, Liu Y, Zhang J. Review: the Role and Mechanisms of Macrophage Autophagy in Sepsis. Inflammation 2019;42:6-19.

22. Dong WW, Zhang YQ, Zhu XY, et al. Protective Effects of Hydrogen-Rich Saline Against LipopolysaccharideInduced Alveolar Epithelial-to-Mesenchymal Transition and Pulmonary Fibrosis. Medical Science Monitor 2017;23:2357-64.

23. Chen J, Zhang H, Hu J, et al. Hydrogen-Rich Saline Alleviates Kidney Fibrosis Following AKI and Retains Klotho Expression. Front Pharmacol 2017;8:499.

24. Du H, Sheng M, Wu L, et al. Hydrogen-Rich Saline Attenuates Acute Kidney Injury After Liver 
Transplantation via Activating p53-Mediated Autophagy. Transplantation 2016;100:563-70.

25. Wang H, Huo X, Chen H, et al. Hydrogen-Rich Saline Activated Autophagy via HIF-1alpha Pathways in Neuropathic Pain Model. Biomed Res Int 2018;2018:4670834.

26. Chen P, Cescon M, Bonaldo P. Autophagy-mediated regulation of macrophages and its applications for cancer. Autophagy 2014;10:192-200.

27. Yang M, Liu JW, Shao JH, et al. Cathepsin S-mediated autophagic flux in tumor-associated macrophages accelerate tumor development by promoting M2 polarization. Mol cancer 2014;13:43.

28. Chen W, Ma T, Shen XN, et al. Macrophage-induced tumor angiogenesis is regulated by the TSC2-mTOR pathway. Cancer Res 2012;72:1363-72.

29. Zhang L, Cardinal JS, Bahar R, et al. Interferon regulatory factor-1 regulates the autophagic response in LPSstimulated macrophages through nitric oxide. Mol Med 2012;18:201-8.

Cite this article as: Qiu P, Liu Y, Chen K, Dong Y, Liu S, Zhang J. Hydrogen-rich saline regulates the polarization and apoptosis of alveolar macrophages and attenuates lung injury via suppression of autophagy in septic rats. Ann Transl Med 2021;9(12):974. doi: 10.21037/atm-21-2489
30. Ryter SW, Mizumura K, Choi AM. The impact of autophagy on cell death modalities. Int J Cell Biol 2014;2014:502676.

31. Liu Y, Levine B. Autosis and autophagic cell death: the dark side of autophagy. Cell Death Differ 2015;22:367-76.

32. Pang J, Peng H, Wang S, et al. Mitochondrial ALDH2 protects against lipopolysaccharide-induced myocardial contractile dysfunction by suppression of ER stress and autophagy. Biochimica et Biophysica Acta (BBA) Molecular Basis of Disease 2019;1865:1627-41.

33. Iketani M, Ohshiro J, Urushibara T, et al. Preadministration of Hydrogen-Rich Water Protects Against Lipopolysaccharide-Induced Sepsis and Attenuates Liver Injury. Shock 2017;48:85-93.

34. Ikeda M, Shimizu K, Ogura H, et al. Hydrogen-Rich Saline Regulates Intestinal Barrier Dysfunction, Dysbiosis and Bacterial Translocation in a Murine Model of Sepsis. Shock 2018;50:640-7.

(English Language Editor: J. Teoh) 